\title{
The predominant expression of cancer stem cell marker ALDH1A3 in tumor infiltrative area is associated with shorter overall survival of human glioblastoma
}

Chao Gan ${ }^{1,2}$, Daniela Pierscianek ${ }^{1}$, Nicolai El Hindy ${ }^{1,3}$, Yahya Ahmadipour ${ }^{1}$, Kathy Keyvani ${ }^{4}$, Ulrich Sure ${ }^{1}$ and Yuan Zhu ${ }^{1 *}$ iD

\begin{abstract}
Background: ALDH1A3 is a cancer stem cell marker in neoplasms including glioblastoma (GBM). However, the comprehensive role of ALDH1A3 in GBM remains unclear. This study attempted to investigate the expression of ALDH1A3 in human GBM tissues and its association with clinical parameters.

Methods: Thirty primary GBM and 9 control were enrolled in this study. ALDH1A3 mRNA and protein expression levels were detected by $\mathrm{RT}^{2}$-PCR and western blot, respectively. Immunohistochemistry and immunofluorescence staining were performed to evaluate the regional and cellular expression manner of ALDH1A3. The association of ALDH1A3 expression with multiple clinical parameters was analyzed.

Results: ALDH1A3 protein level, but not mRNA level, in a subgroup of GBM was significantly higher than that in the control group. ALDH1A3 immunoreactivity was detected heterogeneously in individual GBMs. Fifteen of 30 cases showed a positive of ALDH1A3 immunoreactivity which was predominantly observed in the tumor infiltrative area (TI). Double immunofluorescence staining revealed a co-localization of ALDH1A3 with GFAP in glial-shaped cells and in tumor cells. ALDH1A3 immunoreactivity was often merged with CD44, but not with CD68. Moreover, ALDH1A3 expression was positively associated with the tumor edema grade and inversely with overall survival (OS) (median OS: 16 months vs 10 months), but with neither MGMT promoter methylation status nor Ki67 index in GBM. An upregulation of ALDH1A3 was accompanied by a reduced expression of STAT3 $\beta$ and p-STAT3 $\beta$.

Conclusions: Inter- and intra-tumoral heterogeneous expression of ALDH1A3 was exhibited in GBMs. A high immunoreactivity of ALDH1A3 in tumor infiltrative area was associated with shorter OS, especially in patients with MGMT promoter methylation. Our findings propose ALDH1A3 not only as a predictive biomarker but also as a potential target for personalized therapy of GBM.
\end{abstract}

Keywords: Primary glioblastoma, ALDH1A3, Cancer stem cell marker, Overall survival, Peritumoral edema

\footnotetext{
* Correspondence: yuan.zhu@uk-essen.de

'Department of Neurosurgery and Spine Surgery, University hospital Essen, University of Duisburg-Essen, Hufelandstrasse 55, 45122 Essen, Germany

Full list of author information is available at the end of the article
}

(c) The Author(s). 2020 Open Access This article is licensed under a Creative Commons Attribution 4.0 International License, which permits use, sharing, adaptation, distribution and reproduction in any medium or format, as long as you give appropriate credit to the original author(s) and the source, provide a link to the Creative Commons licence, and indicate if changes were made. The images or other third party material in this article are included in the article's Creative Commons licence, unless indicated otherwise in a credit line to the material. If material is not included in the article's Creative Commons licence and your intended use is not permitted by statutory regulation or exceeds the permitted use, you will need to obtain permission directly from the copyright holder. To view a copy of this licence, visit http://creativecommons.org/licenses/by/4.0/ The Creative Commons Public Domain Dedication waiver (http://creativecommons.org/publicdomain/zero/1.0/) applies to the data made available in this article, unless otherwise stated in a credit line to the data. 


\section{Background}

Glioblastoma (GBM), the most common primary malignant brain tumor in adults, is genetically and histopathologically highly heterogeneous. A median survival period is 15 months despite the advanced treatment including surgical resection and chemoradiotherapy [1]. Increasing evidence suggests that cancer stem cells (CSCs) are crucial for tumorigenesis, therapeutic resistance and recurrence in GBM [2,3]. Given that subventricular zone (SVZ) consists of enriched neural stem cells that possess the capacity to generate neurons and glia throughout adulthood $[4,5]$. Gliomas are thus often presumed being initiated by neural stem cells in SVZ [6-8]. Indeed, expression of multiple CSC markers in GBM is negatively associated with overall survival in GBM patients $[9,10]$. Therefore, targeting CSCs is considered as a promising therapeutic strategy.

Aldehyde dehydrogenases (ALDHs) are a group of enzymes consisting of 19 isoforms. Besides the metabolic functions [11, 12], high ALDH activity is considered as a hallmark of CSCs in various cancers [13]. Targeting ALDH inhibits the proliferation of GBM tumor cells and CSCs [14]. ALDH1A3 is the most upregulated between ALDH high and low subgroups of glioma cells among 19 isoform of ALDH family [15]. ALDH1A3 prominently emerges as a CSC marker to be targeted in multiple neoplasms [16-18]. Of note, ALDH1A3 is enriched in mesenchymal subtype (MES) of GBM patients, thereby being a sensitive and specific marker for MES GBM subtype [15]. ALDH1A3 is crucial for transition from proneural-CSCs to MES-CSC and is important for the maintenance of MES subtype [19]. Besides, ALDH1A3 plays also important roles in regulating self-renewal, differentiation and chemo/radio-resistance [20].

Most of the studies of ALDH1A3 expression in dataset were performed at transcriptional level by microarray in GBM. However, the comprehensive association of ALDH1A3 protein expression with clinical outcome remains elusive. The present study focused on the investigation of ALDH1A3 protein expression in a cohort of GBM patients with emphasis on its regional expression pattern and cellular localization and on its correlation with clinical parameters. We also explored the STAT3 and Akt/PTEN signaling cascades which might be involved in the regulation of ALDH1A3 expression and in its functions in GBM as studied by other entities. Through this study, we anticipate providing a broader perspective on this molecule as a prognostic biomarker as well as a potential therapeutic target for GBM.

\section{Methods}

Patient cohort, magnetic resonance imaging (MRI)-based edema grading and evaluation of the Karnofsky performance index (KPI)

Surgical specimens $(n=30)$ were collected from adult patients with primary GBM who were treated in the
Department of Neurosurgery at our hospital. All enrolled patients were histopathologically diagnosed with primary GBM (WHO grade IV). Surgical specimens from patients who suffered temporal lobe epilepsy and underwent anterior temporal lobe resections without histopathological findings were used as control $(n=9)$.

Tumor edema appears as a region with increased T2 signal intensity outside the gadolinium-enhanced portion. Peritumoral edema is classified into four grades based on preoperative MRI scans [21]. Briefly, grade zero reflects no edema on preoperative scans; if edema is seen as less than, or approximately equal to, or greater than the tumor itself, the edema is graded as I, II, or III, respectively.

The preoperative KPI as one of the major prognostic indicators for GBM survival was used to assess all patients enrolled in the present study.

\section{Evaluation of 06-methylguanine-methyltransferase (MGMT) promoter methylation and IDH1 mutation}

For MGMT promoter methylation analysis, genomic DNA was isolated from paraffin sections of GBM. MGMT promoter methylation was analyzed by methylation-specific PCR as described previously [22].

IDH1-R132H, the most common glioma derived mutation, was determined immuno-histochemically in paraffin-embedded tumor specimens with a specific antibody as described previously [23].

TCGA database analysis of ALDH1A3 gene expression and OS dataset in GBM

The data of ALDH1A3 gene expression and its association with OS in $525 \mathrm{GBM}$ cases and 10 normal control from The Cancer Genome Atlas (TCGA-GBM) were obtained from the GlioVis browser [24]. The gene expression profile was measured using the Affymetrix HT Human Genome U133a microarray platform. KaplanMeier survival curve was generated to show patient survival status between $A L D H 1 A 3$ high and low group with optimal cutoff provided by GlioVis itself.

\section{Real time-reverse transcription-polymerase chain reaction ( $\mathrm{RT}^{2}$-PCR)}

The extraction of total RNA (Qiagen, Hilden) and cDNA synthesis (Bio-Rad, Munich) were performed according to the manufacturer's instructions. The primer sequences and annealing temperatures are listed in Table 1. The following condition was used for real-time PCR: initial denaturation at $95^{\circ} \mathrm{C}$ for $2 \mathrm{~min}, \quad 35-45$ cycles of amplification at $95^{\circ} \mathrm{C}$ for $5 \mathrm{~s}$ and at annealing temperature for $25 \mathrm{~s}$. Melting curve was obtained using the following settings: $95^{\circ} \mathrm{C}$ for $1 \mathrm{~min}$, and $55^{\circ} \mathrm{C}$ for $1 \mathrm{~min}$, and $55-$ $95^{\circ} \mathrm{C}$ with a heating increase rate of $0.5^{\circ} \mathrm{C}$ every $10 \mathrm{~s}$. Relative mRNA expression (fold change) was calculated 
Table 1 Primer sequences and annealing temperatures for realtime reverse-transcription $P C R\left(R T^{2}-P C R\right)$

\begin{tabular}{|c|c|c|}
\hline $\begin{array}{l}\text { Primer } \\
\text { name }\end{array}$ & Sequence & $\begin{array}{l}\text { Annealing temperature } \\
\left({ }^{\circ} \mathrm{C}\right)\end{array}$ \\
\hline Nestin & & 62 \\
\hline for. & $\begin{array}{l}\text { CTCCAAGAATGGAGGCTG } \\
\text { TAGGAA }\end{array}$ & \\
\hline rev. & $\begin{array}{l}\text { CCTATGAGATGGAGCAGG } \\
\text { CAAGA }\end{array}$ & \\
\hline CD133 & & 60 \\
\hline for. & CAGAAGGCATATGAATCC & \\
\hline rev. & CACCACATTTGTTACAGC & \\
\hline CD44 & & 60 \\
\hline for. & CCCAGATGGAGAAAGCTCTG & \\
\hline rev. & ACTTGGCTTTCTGTCCTCCA & \\
\hline$Y K L 40$ & & 63 \\
\hline for. & GACCACAGGCCATCACAGTCC & \\
\hline rev. & $\begin{array}{l}\text { TGTACCCCACAGCATAGTCAGT } \\
\text { GTT }\end{array}$ & \\
\hline ALDH1A3 & & 60 \\
\hline for. & TCTCGACAAAGCCCTGAAGT & \\
\hline rev. & TATTCGGCCAAAGCGTATTC & \\
\hline OLIGL & & 60 \\
\hline for. & СTCCTCAAATCGCATCCAGA & \\
\hline rev. & AGAAAAAGGTCATCGGGCTC & \\
\hline $50 \times 2$ & & 64 \\
\hline for. & $\begin{array}{l}\text { ACCGGCGGCAACCAGAAG } \\
\text { AACAG }\end{array}$ & \\
\hline rev. & GCGCCGCGGCCGGTATTTAT & \\
\hline
\end{tabular}

for. forward, rev. reverse

according to the cycle threshold approach $\left(2^{-\Delta \Delta C T}\right.$ method), and normalized to the reference gene GAPDH as described [25].

\section{Immunohistochemistry staining and analysis}

Immunohistochemistry was performed on formalin-fixed and paraffin-embedded (FFPE) GBM sections $(n=30)$. Briefly, after deparaffinization in gradient ethanol, heatinduced epitope retrieval and blocking the unspecific binding, sections were incubated with rabbit antiALDH1A3 primary antibody (1:250, Novus Biologicals) overnight at $4{ }^{\circ} \mathrm{C}$. After the incubation with a HRPconjugated secondary antibody (1:1000, Cell Signaling Technology), the sections were incubated with the substrate 3,3'-diaminobenzidine kit (Invitrogen) followed by hematoxylin counter staining. Negative control staining was done omitting primary antibody instead of a nonspecific rabbit immunoglobulin fraction (DAKO).

The ALDH1A3 immunoreactivity in all stained sections was quantified according to previous description [26]. Briefly, the intensity of ALDH1A3 immunoreactivity was scored as $0-3: 0=$ negative; $1=$ weak; $2=$ moderate; $3=$ strong. The percentage of positive cells in microscopic images (magnification $\times 200$ ) was counted using ImageJ, and four categories (0-3) were defined: category $0,<1 \%$; category $1,1-5 \%$; category $2,5-10 \%$; category $3,>10 \%$. The immunoreactive score (IRS) was calculated using the following formula: the score of the immuno-intensity $\times$ the score of positive percentage. According to IRS, the patients were classified to three groups: negative/low: IRS $\leq$ 2; medium: IRS $>2$; and high: IRS $\geq 6$.

\section{Double immunofluorescence staining and imaging}

For FFPE tissue sections, deparaffinization, heat-induced epitope retrieval, and blocking steps were performed as described previously [22]. Thereafter, the sections were incubated overnight at $4{ }^{\circ} \mathrm{C}$ in the primary antibody mixtures containing rabbit anti-ALDH1A3 (1:250) and a cell type specific marker antibody, i.e., mouse anti-GFAP (1: 250, Sigma Aldrich) or mouse anti-CD68 (1:100, gift from Neuropathology in our hospital) or rat anti-CD44 (1:100, Invitrogen). The sections were incubated with the mixture of biotinylated goat anti-rabbit IgG and Texas red conjugated horse anti-mouse IgG at room temperature for $1 \mathrm{~h}$ followed by the substrate reaction with FITC-conjugated avidin. Counter staining was done with Hoechst-33,342. Images were acquired using Axio Imager M2 microscope (Zeiss) with the ApoTome. 2 system for optical sections.

\section{Western blot}

Total protein extraction and electrophoresis were performed as before [21]. The primary antibody reaction was done overnight at $4{ }^{\circ} \mathrm{C}$ with the following primary antibodies (excepting ALDH1A3 antibody, all from Cell Signaling Technology): anti-ALDH1A3 (1:1000, Novus Biologicals), anti-STAT3 (1:1000), anti-phospho-STAT3 (Tyr705) (p-STAT3) (1:2000), anti-GAPDH (1:1000), anti-phospho-Akt (Ser437) (p-Akt) (1:1000), anti-PTEN (1:1000). After the second antibody reaction, the blots were incubated with ECL solution (GE Healthcare) and the image was acquired by using ImageQuant LAS 500 (GE Healthcare, Freiburg).

\section{Statistical analysis}

The experimental data were presented as the mean \pm standard deviation (SD). All statistical analysis were performed using the GraphPad Prism 5 and SPSS 23.0. Student $t$ test with Welch's correction was used for data analysis between two groups; one way ANOVA followed by Bonferroni's multiple comparison test was applied for multi-group comparison. The survival curve was plotted using the Kaplan-Meier method and analyzed using logrank test. A $P$ value less than 0.05 was considered statistically significant. 


\section{Results}

Baseline characteristics of patient

The mean age of GBM patients at the first diagnosis was $60.1 \pm 13.3$ years. The ratio of male to female was $1: 1.1$ (14 to16). Among 30 GBMs, 60\% (18/30) patient had a gross total resection of tumor. Twenty-six patients (86.7\%) showed KPI $\geq 70$. Fourteen of 30 cases $(46.7 \%)$ had a positive methylation status of MGMT promoter. A $\mathrm{R} 132 \mathrm{H}$ point mutation of IDH1 was detected in 3 of 20 cases, whereas IDH mutation information was not assessed in the other 10 cases. A standard chemoradiotherapy [1] after surgical resection was applied to $63.3 \%$ $(19 / 30)$ of patients. The mean of OS was $13.3 \pm 9.9$ months. Given that SVZ is suggested as one of the prognostic factors in GBM [6], GBM patients were grouped upon their tumor location, whether the tumor contacted SVZ $(+)$ or not (SVZ-). In 20 of $30(66.7 \%)$ cases GBM tumors were found at the SVZ+ location.

\section{ALDH1A3 mRNA expression was downregulated in GBM: TCGA database and own data}

Microarray-based TCGA database revealed a statistically reduced $A L D H 1 A 3$ mRNA in GBM compared to control group $(P<0.05$, Fig. 1a) and a negative correlation between the ALDH1A3 mRNA and the OS of GBM patients, i.e., higher $A L D H 1 A 3$ mRNA expression and shorter OS with optimal cutoff of $A L D H 1 A 3$ mRNA value at $4.22(P<0.01$, Fig. 1 b) .

To validate the finding from the TCGA datasets, RTPCR was performed and found a significant downregulation of $A L D H 1 A 3$ mRNA expression in our patient cohort $(P<0.05$, Fig. 1c). To compare SVZ+ GBM with SVZ- GBM in the matter of CSCs characteristics, not only $A L D H 1 A 3$ but also some other known CSC markers including Nestin, CD133, CD44, YKL40, OLIG2 and $S O X 2$ were examined. Interestingly, the $A L D H 1 A 3$ mRNA level was 2.59-fold higher in SVZ+ group compared to SVZ-. However, neither ALDH1A3 nor other detected CSC markers showed significant difference between SVZ+ and SVZ- groups (Fig. 1d).

\section{Characterization of regional and cell type specific expression of ALDH1A3 in GBM}

Immunohistochemistry (IHC) revealed a heterogeneous expression of ALDH1A3 in different GBM sections and in the different regions of individual cases. To characterize its expression pattern upon area of focus, we classified tumor regions into three: tumor center (TC), tumor infiltrative area (TI) and tumor distant area (TD) (Fig. 2a, lines). TI showed a gradually lower tumor cell density in comparison to TC, whereas TD exhibited normal cells as well as some scattered tumor cells. An apparent expression of ALDH1A3 was found in 50\% of GBM specimens (15/30). Figure $2 b$ and $c$ were representative of a negative and a positive ALDH1A3 expression case, respectively. In the cases showing ALDH1A3 expression, ALDH1A3 immunoreactivity was dominantly detected in TI (Fig. 2c, d, g-j), whereas scattered ALDH1A3 positive cells were also found in both TC (Fig. 2e) and TD (Fig. 2f). For quantitative analysis of ALDH1A3 expression, IRS was determined upon area of focus in tumor and classified patient cases into three subgroups: negative/low (IRS $\leq 2$ ), medium (IRS $>2$ ) and high (IRS $\geq 6$ ). According to IRS of 30 patient cases, 24, 3 and 3 cases in TC and 15, 4 and 11 in TI were subgrouped, respectively (Fig. 4a), indicating that TI had a significantly higher expression of ALDH1A3 than TC $(P<0,001)$.

We observed ALDH1A3 expression in tumor cells (Fig. 2g, arrows), in outermost layer cells of some glomeruloid tufts (Fig. 2h, arrows), as well as in vessels (Fig. 2i, arrows). Some multiple-nucleus cells with dendritic-like structures (Fig. 2j, arrows) and small cells displaying glial-shaped morphology (Fig. 2j, arrowheads) also appeared ALDH1A3 positive. Of note, ALDH1A3 immunoreactivity was not detected in pseudopalisades (Fig. 2k, arrows) and necrosis centers (Fig. 2k, asterisk) in all investigated sections in this study. There was no apparent ALDH1A3 positive cells in control (Fig. 2l).

In order to identify ALDH1A3 expressing cells, immunofluorescence staining of ALDH1A3 in combination with cell type specific markers was performed. Some glial-shaped cells and tumor cells were found double positive for ALDH1A3 and GFAP (Fig. 3a, b, arrows, respectively). Interestingly, the immunoreactivity of ALDH1A3 and GFAP was merged also in the dendritic-processed cells (Fig. 3c, arrows) and in cells with multiple nuclei (Fig. 3d, arrows). Cells expressing ALDH1A3 are often found positive for a stem cell surface marker CD44 (Fig. 3f, arrows), but not for a macrophage marker CD68 (Fig. 3e, arrowheads).

\section{Correlation of ALDH1A3 expression with clinical parameters}

According to IRS of ALDH1A3 in TI, the cohort of GBM patients was subgrouped as follows: negative/ lower and medium/high expression of ALDH1A3 (Fig. 4a). The association of ALDH1A3 expression with clinical parameters in each subgroup was analyzed and summarized in Table 2. No statistical significance was found for the correlation of ALDH1A3 expression with all these analyzed parameters except OS and peritumoral edema. A higher expression of ALDH1A3 was significantly associated with a shorter median OS $(P<0.01, \quad \mathrm{HR}=3.170,95 \% \mathrm{CI}: 1.328-$ 7.566) (Fig. 4b). The median survival time of patients 

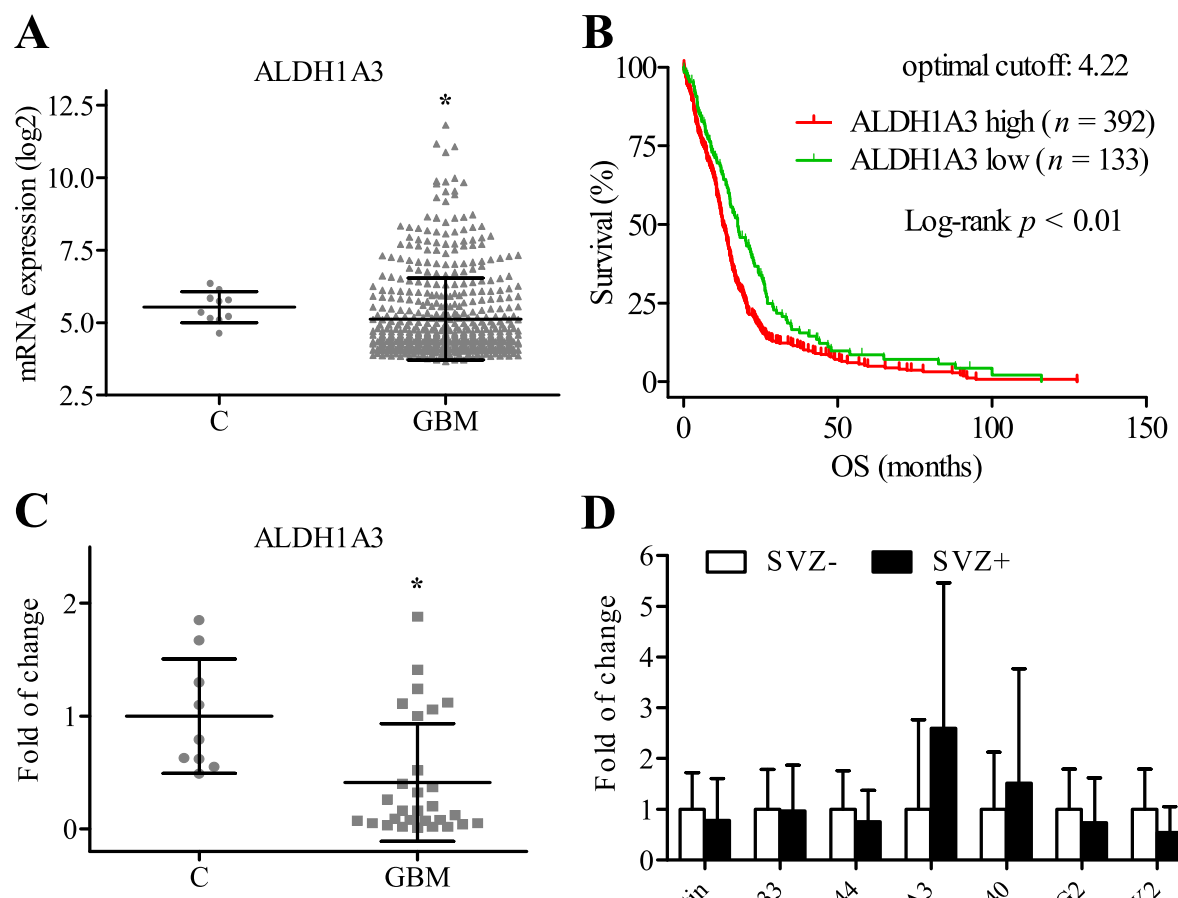

D

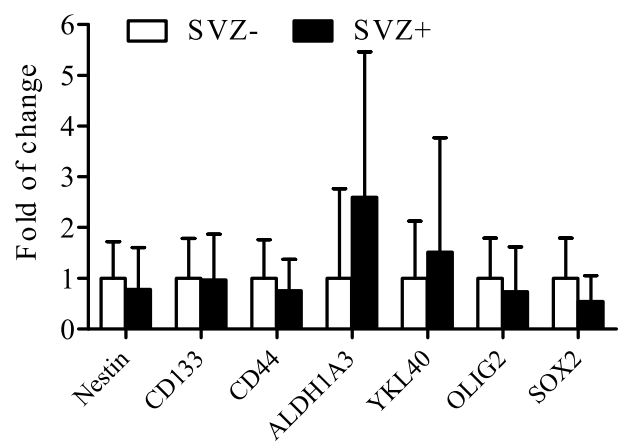

Fig. 1 mRNA expression of ALDH1A3 in GBM. a TCGA database showed a lower expression of ALDH1A3 mRNA in GBM $(n=525)$ compared with the control $(n=10)$. $\mathbf{b}$ The higher expression of ALDHIA3 mRNA was associated with the shorter overall survival (OS) of GBM patients at optimal cutoff based on TCGA database. $\mathbf{c}$ mRNA expression of ALDH1A3 detected in the present study by real-time RT-PCR in primary GBM ( $n=30$ ) and control $(n=9)$ supported the data from TCGA database. $\mathbf{d}$ Subgroup analysis of mRNA expression of ALDH1A3 and other stem cell markers in subventricular zone (SVZ+) and in non-subventricular zone (SVZ-). Student's t test with Welch's correction was utilized between two groups in a, c, $\mathbf{d}\left({ }^{*} P<0.05\right)$

with medium/high ALDH1A3 expression was 10 months, while that of patients with negative/low ALDH1A3 group was 16 months. Furthermore, the higher expression of ALDH1A3 was also associated with a higher grade of peritumoral edema $(P<0.05$, Fig. 4c). We further analyzed the association of MGMT promoter methylation status with OS, and no significant association was found in our patient cohort. The median survival in both MGMT promoter methylation positive (MGMT+, $n=14$ ) and negative (MGMT-, $n=14$ ) patients was 12 months. Next, we also studied association of OS with a combination of MGMT methylation status and ALDH1A3 expression. Of note, the median survival in the subgroup of MGMT+ with low ALDH1A3 IRS was found significantly longer than that in the subgroup of MGMT+ with higher ALDH1A3 IRS (17 months vs 7 months, respectively; $P<0.01$ ). However, there was no significant difference in OS seen between low and higher ALDH1A3 expression in the subgroup of MGMT- patients (13.5 months vs 10.5 months).
In addition, Ki67 index, a proliferation parameter, was 16.3 and $17.3 \%$ in low and higher ALDH1A3 subgroup, respectively (Table 2), and appeared as not associated with ALDH1A3 expression.

\section{Association of the expression of ALDH1A3 and signaling proteins in GBM}

Figure 5a shows representative blots detecting ALDH1A3, STAT3 and p-STAT3, p-Akt, PTEN and GAPDH in GBM and in control. Western blot confirmed distinct expression level of ALDH1A3 in subgroups of GBMs $(P<0.01)$. As expected, an upregulation of ALDH1A3 was confirmed in the GBM cases with higher IRS (IRS $>2$, lane 6-9), whereas a low protein level of ALDH1A3 was detected in the GBM cases with lower IRS (IRS $\leq 2$, lane $3-5)$. Semi-quantitative analysis of the blots revealed a significant upregulation of p-STAT3 $\alpha$, p-STAT3 $\beta$ and p-Akt accompanied by a downregulation of PTEN in GBMs compared to control. Interestingly, the expression of STAT3 $\beta(P<0.01)$ and $\mathrm{p}$-STAT3 $\beta$ $(P<0.05)$, but not STAT3 $\alpha$ and p-STAT3 $\alpha$, was 


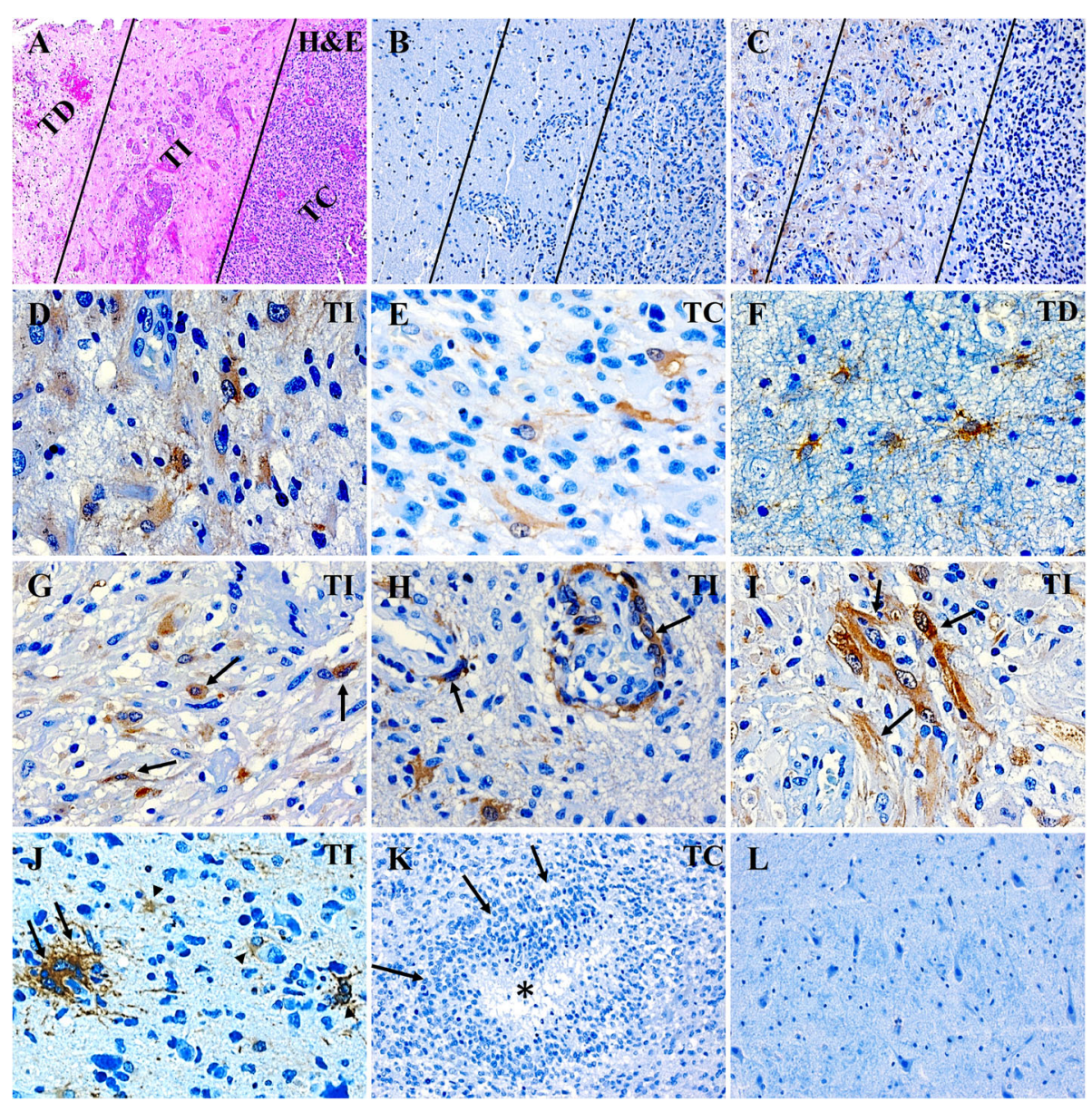

Fig. 2 Immunohistochemistry of ALDH1A3 in GBM. a H\&E staining defined the different regions in GBM sections. TC: tumor center; TI: tumor infiltrative area; and TD: tumor distant area. $\mathbf{b}, \mathbf{c}$ Immunostaining of ALDH1A3 in GBM sections revealed heterogeneous expression of ALDH1A3 in different GBM patient sections $(n=30)$. The representative photos show the absence of ALDH1A3 expression $\mathbf{b}$ and the positive expression of ALDH1A3 c, respectively, in two GBM cases. The positive ALDH1A3 expressing cells were mainly detected in Tl, and much less in TC. $\mathbf{d}$-f Images are representative of the expression of ALDH1A3 in the different regions of GBM tissue. $\mathbf{g}-\mathbf{k}$ Expression of ALDH1A3 in typical tumor cells (arrows in $\mathbf{g}$ ), in out-layer of glomeruloid tufts (arrows in $\mathbf{h}$ ), in some vessels (arrows in $\mathbf{i}$ ), in multi-nuclear cells (arrow in $\mathbf{j}$ ) and in glial-shaped cells (arrowheads in j). ALDH1A3 immunoreactivity was not detected in the necrosis core (asterisk in $\mathbf{k}$ ) and the pseudopalisade structure (arrows in $\mathbf{k}$ ). I ALDH1A3 immunoreactivity was not detected in the non-tumoral control brain section. a-c, original magnification $\times 100$; $\mathbf{d}$-j, original magnification $\times 400$; $\mathbf{k}-\mathbf{l}$, original magnification $\times 200$

inversely associated with the protein level of ALDH1A3 in two subgroups of GBM (Fig. 5b).

\section{Discussion}

Increasing evidence indicates ALDH1A3 as an important molecule that influences a diverse range of biological processes in CSCs and in tumor cells, thereby being associated with the initiation, progression, and prognosis of various cancers including GBM [27]. The present study investigated the expression pattern of ALDH1A3 at both mRNA and protein levels in human GBM specimens with emphasis on its association with clinical parameters of the patients.

Analysis of the microarray based TCGA-GBM dataset revealed that the mean level of $A L D H 1 A 3$ mRNA in the total cohort was lower than that in control. However, when considered in genomic subtypes of GBM [28, 29], its mRNA level appeared significantly higher in the MES than in the classical and proneural subtypes; even in the MES of GBM, the mean level of ALDH1A3 mRNA was comparable with that in control (Supplementary Fig. S2). On the other hand, the TCGA-GBM dataset showed an inverse association of $A L D H 1 A 3$ mRNA expression with OS of GBM. These findings from the TCGA data encouraged us to further investigate implication of ALDH1A3 in GBM. In the present study, we demonstrated a significant downregulation of ALDH1A3 mRNA expression in our GBM cohort in comparison to control, consistent with the findings derived from the TCGA dataset. As the cellular, biological functions of a 

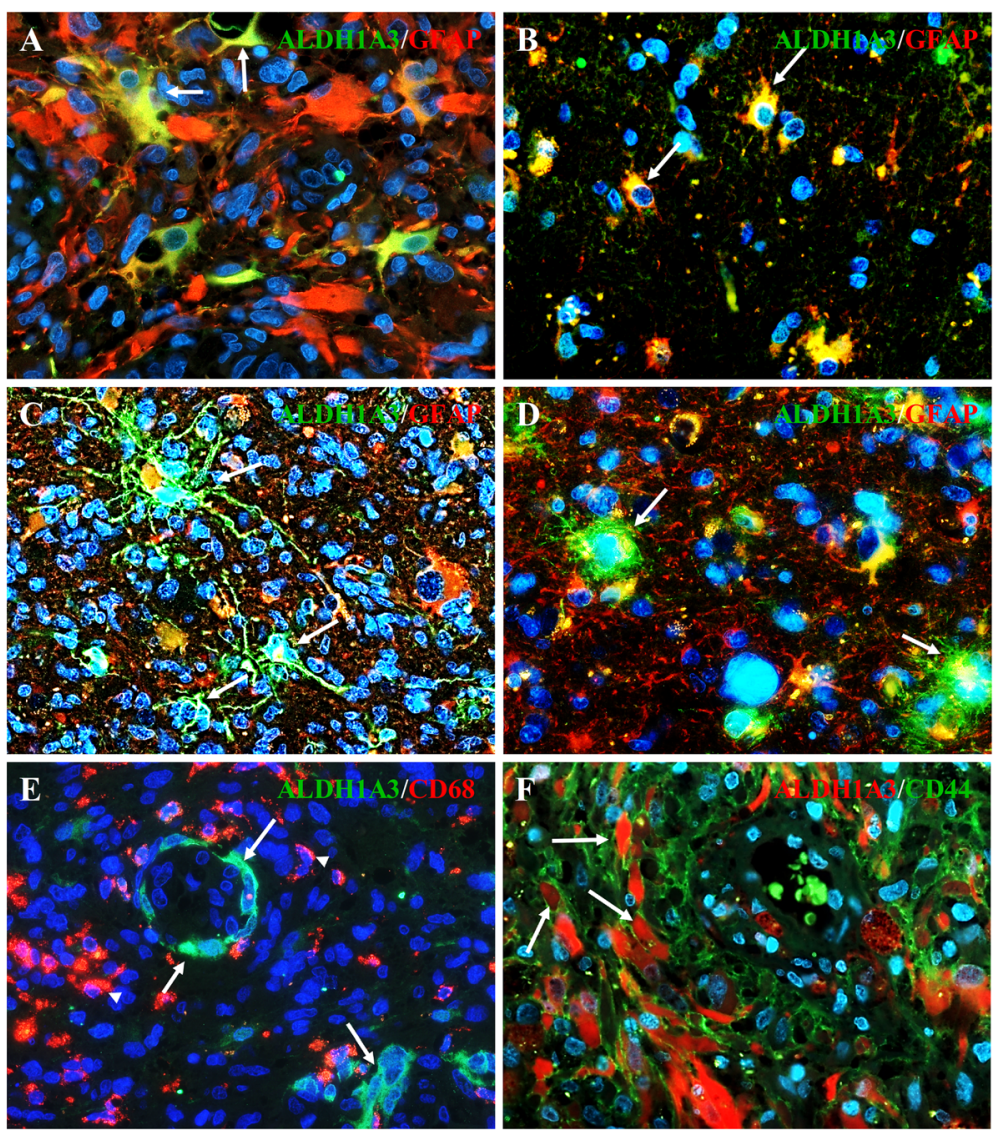

Fig. 3 Double staining of ALDH1A3 with different markers in GBM sections. a-d Double staining of ALDH1A3 with GFAP. In both TC and TI, the co-expression of ALDH1A3 (green) and GFAP (red) was detected in some glial-shaped cells (arrows in a), tumor cells (arrows in $\mathbf{b}$ ) and multinucleated cells (arrows in $\mathbf{c}$ and $\mathbf{d}$ ). e Double staining of ALDH1A3 with CD68. ALDH1A3 immunoreactivity (green) detected in the outer layer of glomeruloid tuft (arrows in e) was negative for the macrophage marker CD68 (red, arrowheads). f Double staining revealed a coexpression (arrows) of ALDH1A3 (red) with the stem cell marker CD44 (green). a-f, original magnification $\times 400$

molecule are ultimately determined by its translational/ post-translational levels, not by its transcriptional level, we next focused on ALDH1A3 expression at protein levels in individual GBM tissues of our cohort. As shown in Fig. 2l, the immunoreactivity of ALDH1A3 was not detected in the control (normal) brain tissue. Furthermore, by western blot we also demonstrated a 4.66 -fold higher protein expression of ALDH1A3 in the subgroup of GBMs with IRS $>2$ than that in the control group (Fig. 5b). These findings allow us to postulate that a higher mRNA level of ALDH1A3 does not necessarily refer to its higher protein level. Indeed, several potential mechanisms regulating the translational and posttranslation of ALDH1A3 have been identified: the hypermethylation status of ALDH1A3 promoter predicts a low expression of ALDH1A3 protein accompanied by a better prognosis of GBM patients [30]; USP9X-mediated deubiquitinase plays an important role in ALDH1A3 protein stabilization [31]; temozolomide (TMZ) treatment at high concentrations does not alter ALDH1A3
mRNA levels, but protein levels through autophagy [32]. Thus, it is more reliable to analyze the association of clinical parameters with ALDH1A3 protein levels.

GBM tumor is composed of heterogeneous cell populations including a small population, glioma CSCs [2], which is related to therapeutic resistance of GBM [3, 33]. As the subventricular zone (SVZ) is often proposed to be the source of CSCs $[6,8]$, we evaluated mRNA expression levels of $A L D H 1 A 3$ as well as other well-known CSC markers including Nestin, CD133, CD44, YKL40, OLIG2, SOX2 in GBM. Despite a 2.5 -fold higher $A L D H 1 A 3$ mRNA detected in the SVZ+ group, none of these investigated CSC markers showed a statistically significant alteration in mRNA levels (Fig. 1d). These results are consistent with previous reports [34, 35], the mechanism under which need to be further investigated.

The ALDH superfamily is the most important aldehyde metabolic enzyme family in human cells and has been linked to metabolism reprogramming in the 


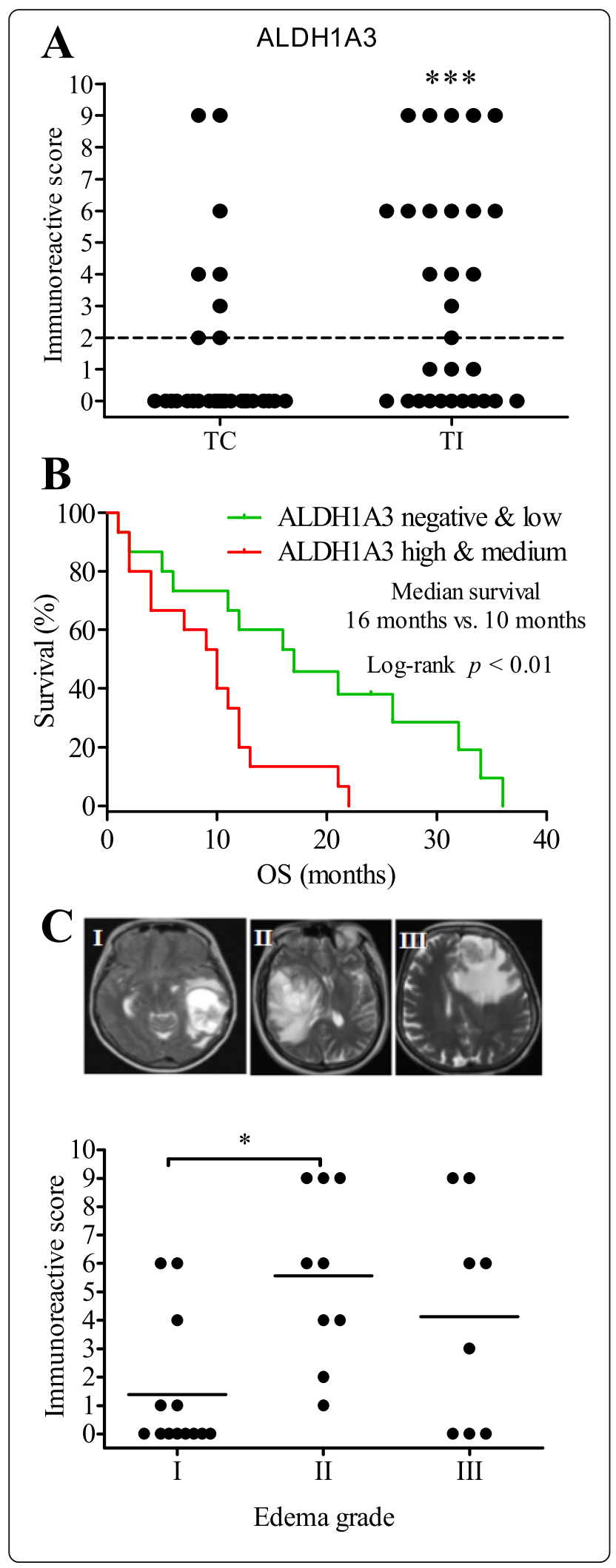

Fig. 4 Association of ALDH1A3 expression with clinical parameters. a Quantitative analysis of ALDH1A3 immunoreactivity in TC and in TI of GBMs. The immunoreactive score (IRS) was evaluated in TC and TI as described in the method $(n=30)$. Based on IRS, TI had a significantly higher ALDH1A3 expression than TC $(P<0.001)$. In according to TI, GBM with IRS $\leq 2$ was grouped as negative/low ( $n=$ 15), whereas GBM with IRS $>2$ was defined as high and medium group $(n=15)$. $\mathbf{b}$ The association of ALDH1A3 expression with the OS period of patients. A higher expression of ALDH1A3 was significantly associated with a shorter OS time. c The representative MRI images show the different grades of edema. Peritumoral edema grade II had a higher IRS of ALDH1A3 compared with edema grade I. Paired t test was performed to compare IRS between TI and TC in a $\left.{ }^{* * *} P<0.001\right)$. One way ANOVA followed by Bonferroni's multiple comparison test, comparing each column to all the other columns, was employed for multi-groups comparison in $\mathbf{c}\left({ }^{*} P<0.05\right)$

initiation, metastasis, and recurrence of cancer [36]. Among the 19 members, the ALDH1A3 isoform has been served as the major source of total ALDH activity in GBM [15]. Thus, it is important to check not only ALDH1A3 mRNA levels but also its protein levels that is more relevant to its enzymatic activity. Our immunohistochemistry study revealed a distinct expression of ALDH1A3 in individual GBM patients and a high intertumoral heterogeneity. A clear positive immunoreactivity of ALDH1A3 was detected only in 15 of 30 (50\%) GBMs. Among these positive cases, 4 and 11 cases showed medium (IRS $>2$ ) and high (IRS $\geq 6$ ) expression of ALDH1A3, respectively. ALDH1A3 positive cells were mostly located at the tumor infiltrative area, suggesting that ALDH1A3 may participate in tumor cell invasiveness and metastasis. In fact, knockdown of ALDH1A3 expression in vitro models also suppressed cancer cell invasion in different entities [37, 38]. We demonstrated that a higher ALDH1A3 IRS was significantly associated with a short OS. As supporting, the expression of ALDH1A3 was positively associated with the grade of peri-tumoral edema that is also a prognostic parameter for GBM patients [39, 40]. Regardless of treatment, MGMT promoter methylation is an independent and favorable prognostic factor in GBM [41]. MGMT encodes a DNA-repair protein that inhibits the effect of treatment through removing alkyl groups from guanine, a target site for alkylating chemotherapy agents such as TMZ. MGMT promoter methylation is associated with higher therapeutic effect of TMZ and longer OS in GBM patients [42]. However, controversial results are also observed in several studies [43, 44]. In the present study, MGMT promoter methylation status was not associated with OS in GBM. Interestingly, when combining MGMT and ALDH1A3 expression together, low expression of ALDH1A3 may sensitively predict a better prognosis than those with higher expression of ALDH1A3 in the subgroup of MGMT+ patients. Hence, evaluation of ALDH1A3 expression might be a powerful 
Table 2 Analysis of the association of ALDH1A3 IRS with clinical parameters

\begin{tabular}{|c|c|c|c|c|}
\hline \multirow[t]{2}{*}{ Clinical parameter } & \multirow{2}{*}{$\begin{array}{l}\text { Patient } \\
\text { number }\end{array}$} & \multicolumn{2}{|l|}{ ALDH1A3 } & \multirow[t]{2}{*}{$P$} \\
\hline & & $\begin{array}{l}\text { negative/low } \\
(\text { IRS } \leq 2)\end{array}$ & $\begin{array}{l}\text { medium/high } \\
(\text { IRS }>2)\end{array}$ & \\
\hline Total patients & 30 & 15 & 15 & \\
\hline Age & & & & $0,456^{a}$ \\
\hline$<60$ & 12 & 7 & 5 & \\
\hline$\geq 60$ & 18 & 8 & 10 & \\
\hline Gender & & & & $0,464^{a}$ \\
\hline Male & 14 & 6 & 8 & \\
\hline Female & 16 & 9 & 7 & \\
\hline KPI & & & & $1,000^{b}$ \\
\hline$\geq 70$ & 26 & 13 & 13 & \\
\hline$<70$ & 4 & 2 & 2 & \\
\hline SVZ & & & & $0,121^{a}$ \\
\hline+ & 20 & 12 & 8 & \\
\hline- & 10 & 3 & 7 & \\
\hline Extent of Resection & & & & $0,456^{\mathrm{a}}$ \\
\hline GTR & 18 & 8 & 10 & \\
\hline Partial & 12 & 7 & 5 & \\
\hline $\begin{array}{l}\text { MGMT promotor } \\
\text { methylation }\end{array}$ & & & & $0,464^{\mathrm{a}}$ \\
\hline Yes & 14 & 6 & 8 & \\
\hline No & 14 & 9 & 7 & \\
\hline N/A & 2 & 1 & 1 & \\
\hline IDH1 mutation & & & & $0,270^{b}$ \\
\hline Yes & 3 & 2 & 1 & \\
\hline No & 17 & 5 & 12 & \\
\hline N/A & 10 & 8 & 2 & \\
\hline $\begin{array}{l}\text { Standard } \\
\text { chemoradiotherapy }\end{array}$ & & & & $0,256^{a}$ \\
\hline Yes & 19 & 11 & 8 & \\
\hline No & 11 & 4 & 7 & \\
\hline Ki67 index (\%) & 27 & $\begin{array}{l}16,3 \pm 3,1 \\
(n=12)\end{array}$ & $\begin{array}{l}17,3 \pm 2,0 \\
(n=15)\end{array}$ & $0,789^{\circ}$ \\
\hline
\end{tabular}

IRS immunoreactive score, KPI Karnofsky Performance Index, SVZ +/- tumor contacted to subventricular zone (SVZ+) or contacted to non-subventricular zone (SVZ-), GTR gross total resection, MGMT O6-methylguaninemethyltransferase, IDH1 isocitrate dehydrogenase 1, N/A not applicable ${ }^{\mathrm{a}}$ Pearson chi-square test; ${ }^{\mathrm{b}}$ Fisher's exact test; ${ }^{\mathrm{C}}$ Student $\mathrm{t}$ test with Welch's correction

prognostic tool in combination with MGMT promoter methylation status. IDH1 mutation is another prognostic maker for GBM. In TCGA database ALDH1A3 mRNA expression is negatively associated with IDH1 mutation [15]. In our cohort only 3 of 30 patients were identified with IDH1 mutation, and nevertheless, we did analysis of the association of ALDH1A3 expression with IDH1 mutation status. As predicted, no association was found between the level of ALDH1A3 protein or mRNA with

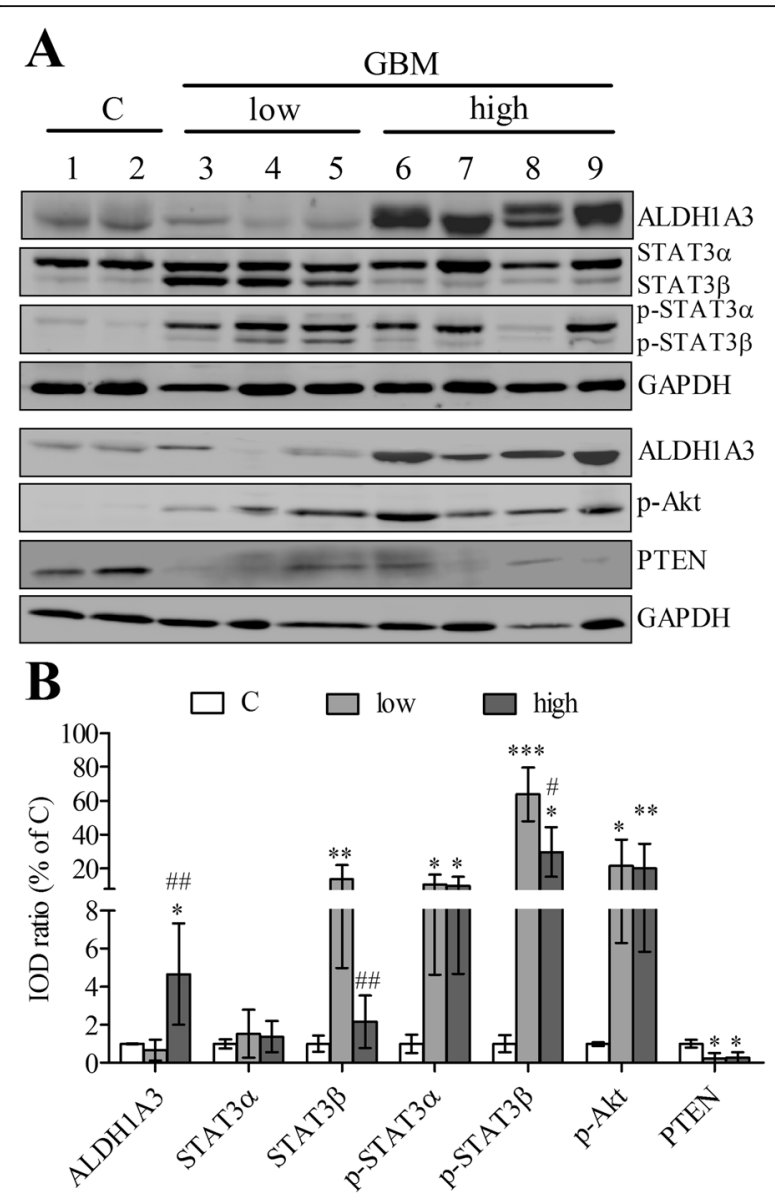

Fig. 5 Detection of ALDH1A3 and the signaling proteins in GBM by western blot. a Representative blots detected the expression of STAT3 and p-STAT3, p-Akt, PTEN and GAPDH in the control (C, lane $1-2$ ) and in GBM cases with lower immunoreactivity score (IRS $\leq 2$, lane 3-5) and with higher immunoreactivity score (IRS $>2$, lane 6-9) (See original images in Supplementary Fig. S1). b Semi-quantification of the blots. The integrated optical density (IOD) of the individual bands on blots was measured by Image and the ratio of the target protein to the housekeeping protein GAPDH was calculated. The relative expression data were presented when the ratio of the control group was normalized as 1. One way ANOVA followed by Bonferroni's multiple comparison test was employed for multigroups comparison in $\mathbf{b}$. ${ }^{*} P<0.05$, ${ }^{* *} P<0.01$ and ${ }^{* *} P<0.001$ : compared with the control (C); \#P<0.05 and \#\#P<0.01, compared with the group "low" (IRS $\leq 2)$

IDH1 mutation. Therefore, a larger patient cohort is needed to verify the association of ALDH1A3 protein with IDH1 mutation in the future. Finally, we also observed the expression of ALDH1A3 frequently in the outer layer of different stage of glomeruloid tufts and in some endothelial cells of tumor vessels. In line with the pro-angiogenic functions of ALDH1A3 in vitro [45, 46], our findings by tissue staining also implicate ALDH1A3 in microvascular proliferation and in neo-angiogenesis in a subgroup of GBM. These clinical associations highlight ALDH1A3 as a potential prognostic biomarker and 
as a therapeutic target preventing tumor invasion and angiogenesis.

The TI consists of various cell types including infiltrating tumor cells, CSCs, reactive astrocyte, microglia, oligodendrocytes, inflammatory and immune cells, endothelial cells, and stromal cells thereby creating a complex microenvironment feasible for tumor growth and invasion as well as survival and therapy-resistance $[47,48]$. To identify a cell type expressing ALDH1A3, we performed double staining of ALDH1A3 with the following cell type specific markers: GFAP, CD68 and CD44. Among ALDH1A3 positive cells, there were some GFAP positive cells likely reactive astrocytes or tumor cells due to their distinct morphology. As the coexpression of ALDH1A3 with the stem cell marker CD44 was also found, we propose that ALDH1A3 positive tumor cells might possess the stem cell-like properties involved in tumor progression and therapy resistance. Tumor-associated macrophages (TAMs) are highlighted in GBM due to the considerable size of their population (30-50\%) [49]. But, the present study did not observe cells co-expressing ALDH1A3 and CD68. The possible implication of ALDH1A3 in immunoreaction mediated by immune cells needs to be further studied.

Along with important roles of ALDH1A3 in cancer and cancer stem cells, its underlying regulatory mechanism has become of interest. Several non-coding microRNAs including miR-600hg [50], miR-7 [51], miR-187 and $\mathrm{miR}-125 \mathrm{a} / \mathrm{b}$ [27] have been identified to recognize ALDH1A3 as a target gene and to suppress ALDH1A3 expression. Forkhead Box D1 (FOXD1) regulates the transcriptional activity of ALDH1A3 in glioma stem cells (GSCs) of MES subtype [52]. The hedgehog pathway can significantly increase the expression of ALDH1 in cancer stem cells of ovary, which can also be a possible mechanism in GBM [53]. In a recent study, ALDH1A3 is stabilized upon ubiquitin-specific protease 9X (USP9X) in the MES GSCs. In contrast, deletion of USP9X induces degradation of ALDH1A3, and a subsequent decrease in p-STAT3, whereas overexpression of ALDH1A3 can restore p-STAT3 expression in GSCs in which downregulation of ALDH1A3 was induced by USP9X depletion [31]. On the other hand, in NSCLC CSCs, activation of STAT3 pathway significantly increases ALDH1A3 expression while multiple inhibitors of STAT3 signaling can decreased ALDH1A3 expression [17]. Taken together, we assume that there might be a feedback loop between ALDH1A3 and STAT3 to promote tumor progression in cancers. Developing STAT3 signaling inhibitors has been a new strategy for anticancer therapy. However, so far none of them is satisfied in the clinic studies [54]. STAT3 has two mainly isoforms, STAT3 $\alpha$ and STAT3 $\beta$, of which the latter is a suppressive factor of STAT3 $\alpha$ due to lacking the transactivation domain
[55]. In the present study, we found that ALDH1A3 protein expression was inversely associated with the levels of total STAT3 $\beta$ and p-STAT3 $\beta$ but no association was seen between the expression of ALDH1A3 and STAT3 $\alpha$. The association and the underlying regulatory mechanism of ALDH1A3 and STAT3 in GBM need to be further elucidated by using gene techniques in the future. Akt/PTEN is an important signaling implicated in numerous malignant tumors including GBM. We demonstrated a significant increase in p-Akt levels concomitantly accompanied by a downregulation of PTEN in GBM. However, the level of PTEN/p-Akt did not appear to be associated with ALDH1A3 expression in GBMs, suggesting that PTEN/Akt may not be a direct downstream signaling cascade trigged by ALDH1A3.

\section{Conclusion}

The present study revealed a high intra- and intertumoral heterogeneity of ALDH1A3 expression manner in GBM. ALDH1A3 expression enriched in tumor infiltrative region highlights its crucial role in tumor invasiveness and progression. Moreover, we demonstrated an inverse association of ALDH1A3 expression with the prognosis of GBM, supporting ALDH1A3 as a prognostic marker and as a potential target for future GBM therapy.

\section{Supplementary information}

Supplementary information accompanies this paper at https://doi.org/10 1186/s12885-020-07153-0.

Additional file 1: Supplementary Figure S1. Original blots of western blotting. Supplementary Figure S2. ALDH1A3 mRNA expression in subtypes of GBM and normal control based on TCGA database. Among three subtypes of GBM, ALDH1A3 mRNA level in the classical and proneural subtypes of GBM was significantly lower than that in the control and in the mesenchymal GBM. Student $t$ test with Welch's correction was used for data analysis between subgroups. ${ }^{* *} P<0.01$, ${ }^{* * *} P<0.001$, compared with control; \#\#\#P<0.001, compared with mesenchymal.

\section{Abbreviations}

ALDH1A3: Aldehyde dehydrogenase 1 family, member A3; ALDHs: Aldehyde dehydrogenases; CSCs: Cancer stem cells; FFPE: Formalin-fixed and paraffinembedded; GAPDH: Glyceraldehyde 3-phosphate dehydrogenase;

GBM: Glioblastoma; GFAP: Glial fibrillary acidic protein; IDH1: Isocitrate dehydrogenase 1; IRS: Immunoreactive score; KPI: Karnofsky performance index; MES: Mesenchymal subtype; MGMT: O6-methylguaninemethyltransferase; MRI: Magnetic resonance imaging; OS: Overall survival; PTEN: Phosphatase and tensin homolog; $\mathrm{RT}^{2}$-PCR: Real time reverse transcriptase polymerase chain reaction; STAT3: Signal transducer and activator of transcription 3; SVZ: Subventricular zone; TAMs: Tumor-associated macrophages; TC: Tumor center; TCGA: The cancer genome atlas; TD: Tumor distant area; TI: Tumor infiltrative area; TMZ: Temozolomide

\section{Acknowledgements}

We thank Ms. Rita Haase and Ms. Michaela Hiber for their technical assistance. CG received a scholarship from the Medical Faculty, University of Duisburg-Essen. We also thank Dr. Su Na Kim for careful proofreading of the manuscript. 


\section{Authors' contributions}

CG contributed to the main experiments, data collection and analysis and participated in the manuscript preparation; DP, YA and NEH: clinical data collection and analysis; KK: histopathological diagnosis of GBM, tissue sectioning and immunostaining; US and YZ: the study conception, design and manuscript preparation. All authors have read and approved the final manuscript.

\section{Funding}

This study was supported financially by the IFORES-program at the Medical Faculty, University of Duisburg-Essen to Y.Z. The faculty paid the material necessary to elaborate the research work. Open access funding provided by Projekt DEAL.

\section{Availability of data and materials}

All the data generated or analyzed during this study are included in this article.

\section{Ethics approval and consent to participate}

This study was strictly performed in accordance with the Declaration of Helsinki and approved by the local ethics committee of the University Hospital Essen. Informed writing consent was obtained from all the patients before the sample collection.

\section{Consent for publication}

Not applicable.

\section{Competing interests}

The authors declare that they have no competing interests.

\section{Author details}

'Department of Neurosurgery and Spine Surgery, University hospital Essen, University of Duisburg-Essen, Hufelandstrasse 55, 45122 Essen, Germany. ${ }^{2}$ Department of Neurosurgery, Tongji Hospital, Tongji Medical College, Huazhong University of Science and Technology, Wuhan, China. ${ }^{3}$ Present Address: Department of Spine- and Peripheral Nerve-Surgery, St. Christophorus 625 Hospital, Werne, Germany. ${ }^{4}$ Institute of Neuropathology, University hospital Essen, University of Duisburg-Essen, Essen, Germany.

\section{Received: 26 May 2020 Accepted: 8 July 2020}

Published online: 17 July 2020

\section{References}

1. Stupp R, Mason WP, van den Bent MJ, Weller M, Fisher B, Taphoorn MJ, et al. Radiotherapy plus concomitant and adjuvant temozolomide for glioblastoma. N Engl J Med. 2005;352(10):987-96.

2. Sharifzad F, Ghavami S, Verdi J, Mardpour S, Mollapour Sisakht M, Azizi Z, et al. Glioblastoma cancer stem cell biology: potential theranostic targets. Drug Resist Updat. 2019;42:35-45.

3. Lathia JD, Mack SC, Mulkearns-Hubert EE, Valentim CL, Rich JN. Cancer stem cells in glioblastoma. Genes Dev. 2015;29(12):1203-17.

4. Alvarez-Buylla A, Garcia-Verdugo JM. Neurogenesis in adult subventricular zone. J Neurosci. 2002;22(3):629-34.

5. Doetsch F, Garcia-Verdugo JM, Alvarez-Buylla A. Cellular composition and three-dimensional organization of the subventricular germinal zone in the adult mammalian brain. J Neurosci. 1997;17(13):5046-61.

6. Lim DA, Cha S, Mayo MC, Chen MH, Keles E, VandenBerg S, et al. Relationship of glioblastoma multiforme to neural stem cell regions predicts invasive and multifocal tumor phenotype. Neuro-Oncology. 2007;9(4):424-9.

7. Qin EY, Cooper DD, Abbott KL, Lennon J, Nagaraja S, Mackay A, et al. Neural Precursor-Derived Pleiotrophin Mediates Subventricular Zone Invasion by Glioma. Cell. 2017;170(5):845-59 e19.

8. Lee JH, Lee JE, Kahng JY, Kim SH, Park JS, Yoon SJ, et al. Human glioblastoma arises from subventricular zone cells with low-level driver mutations. Nature. 2018;560(7717):243-7.

9. Ulasov IV, Mijanovic O, Savchuk S, Gonzalez-Buendia E, Sonabend A, Xiao T, et al. TMZ regulates GBM stemness via MMP14-DLL4-Notch3 pathway. Int J Cancer. 2020;146(8):2218-28.

10. Hou C, Ishi Y, Motegi H, Okamoto M, Ou Y, Chen J, et al. Overexpression of CD44 is associated with a poor prognosis in grade II/III gliomas. J NeuroOncol. 2019;145(2):201-10.
11. Jackson B, Brocker C, Thompson DC, Black W, Vasiliou K, Nebert DW, et al. Update on the aldehyde dehydrogenase gene (ALDH) superfamily. Hum Genomics. 2011;5(4):283-303.

12. Marchitti SA, Brocker C, Stagos D, Vasiliou V. Non-P450 aldehyde oxidizing enzymes: the aldehyde dehydrogenase superfamily. Expert Opin Drug Metab Toxicol. 2008:4(6):697-720.

13. Moreb JS. Aldehyde dehydrogenase as a marker for stem cells. Curr Stem Cell Res Ther. 2008;3(4):237-46.

14. Kuo YC, Wang $L$, Rajesh R. Targeting human brain cancer stem cells by curcumin-loaded nanoparticles grafted with anti-aldehyde dehydrogenase and sialic acid: Colocalization of ALDH and CD44. Mater Sci Eng C Mater Biol Appl. 2019;102:362-72.

15. Li G, Li Y, Liu X, Wang Z, Zhang C, Wu F, et al. ALDH1A3 induces mesenchymal differentiation and serves as a predictor for survival in glioblastoma. Cell Death Dis. 2018;9(12):1190.

16. Vidovic D, Huynh TT, Konda P, Dean C, Cruickshank BM, Sultan M, et al. ALDH1A3-regulated long non-coding RNA NRAD1 is a potential novel target for triple-negative breast tumors and cancer stem cells. Cell Death Differ. 2020;27(1):363-78,

17. Shao C, Sullivan JP, Girard L, Augustyn A, Yenerall P, Rodriguez-Canales J, et al. Essential role of aldehyde dehydrogenase $1 \mathrm{~A} 3$ for the maintenance of non-small cell lung cancer stem cells is associated with the STAT3 pathway. Clin Cancer Res. 2014;20(15):4154-66.

18. Perez-Alea M, McGrail K, Sanchez-Redondo S, Ferrer B, Fournet G, Cortes J, et al. ALDH1A3 is epigenetically regulated during melanocyte transformation and is a target for melanoma treatment. Oncogene. 2017; 36(41):5695-708.

19. Mao P, Joshi K, Li J, Kim SH, Li P, Santana-Santos L, et al. Mesenchymal glioma stem cells are maintained by activated glycolytic metabolism involving aldehyde dehydrogenase 1A3. Proc Natl Acad Sci U S A. 2013. 110(21):8644-9.

20. Durinikova E, Kozovska Z, Poturnajova M, Plava J, Cierna Z, Babelova A, et al. ALDH1A3 upregulation and spontaneous metastasis formation is associated with acquired chemoresistance in colorectal cancer cells. BMC Cancer. 2018; 18(1):848

21. Lambertz N, El Hindy N, Kreitschmann-Andermahr I, Stein KP, Dammann P, Oezkan N, et al. Downregulation of programmed cell death 10 is associated with tumor cell proliferation, hyperangiogenesis and peritumoral edema in human glioblastoma. BMC Cancer. 2015;15:759.

22. El Hindy N, Keyvani K, Pagenstecher A, Dammann P, Sandalcioglu IE, Sure U, et al. Implications of DII4-notch signaling activation in primary glioblastoma multiforme. Neuro-Oncology. 2013;15(10):1366-78.

23. Ahmadipour Y, Jabbarli R, Gembruch O, Pierscianek D, Darkwah Oppong M, Dammann $P$, et al. Impact of Multifocality and molecular markers on survival of Glioblastoma. World Neurosurg. 2019;122:e461-e6.

24. GlioVis: TCGA_GBM dataset. http://gliovis.bioinfo.cnio.es/. Accessed 20 Feb 2020.

25. Livak KJ, Schmittgen TD. Analysis of relative gene expression data using real-time quantitative PCR and the 2(-Delta Delta $C(T))$ method. Methods. 2001;25(4):402-8.

26. Krajewska M, Krajewski S, Epstein JI, Shabaik A, Sauvageot J, Song K, et al. Immunohistochemical analysis of bcl-2, bax, bcl-X, and mcl-1 expression in prostate cancers. Am J Pathol. 1996;148(5):1567-76.

27. Duan JJ, Cai J, Guo YF, Bian XW, Yu SC. ALDH1A3, a metabolic target for cancer diagnosis and therapy. Int J Cancer. 2016;139(5): $965-75$.

28. Verhaak RG, Hoadley KA, Purdom E, Wang V, Qi Y, Wilkerson MD, et al. Integrated genomic analysis identifies clinically relevant subtypes of glioblastoma characterized by abnormalities in PDGFRA, IDH1, EGFR, and NF1. Cancer Cell. 2010;17(1):98-110.

29. Wang Q, Hu B, Hu X, Kim H, Squatrito M, Scarpace L, et al. Tumor Evolution of Glioma-Intrinsic Gene Expression Subtypes Associates with Immunological Changes in the Microenvironment. Cancer Cell. 2017;32(1): 42-56 e6.

30. Zhang W, Yan W, You G, Bao Z, Wang Y, Liu Y, et al. Genome-wide DNA methylation profiling identifies ALDH1A3 promoter methylation as a prognostic predictor in G-CIMP- primary glioblastoma. Cancer Lett. 2013; 328(1):120-5.

31. Chen Z, Wang HW, Wang S, Fan L, Feng S, Cai X, et al. USP9X deubiquitinates ALDH1A3 and maintains mesenchymal identity in glioblastoma stem cells. J Clin Invest. 2019;129(5):2043-55. 
32. Wu W, Schecker J, Wurstle S, Schneider F, Schonfelder M, Schlegel J. Aldehyde dehydrogenase 1A3 (ALDH1A3) is regulated by autophagy in human glioblastoma cells. Cancer Lett. 2018;417:112-23.

33. Kahlert UD, Joseph JV, Kruyt FAE. EMT- and MET-related processes in nonepithelial tumors: importance for disease progression, prognosis, and therapeutic opportunities. Mol Oncol. 2017:11(7):860-77.

34. Kappadakunnel M, Eskin A, Dong J, Nelson SF, Mischel PS, Liau LM, et al. Stem cell associated gene expression in glioblastoma multiforme: relationship to survival and the subventricular zone. J Neuro-Oncol. 2010;96(3):359-67.

35. Jungk C, Mock A, Exner J, Geisenberger C, Warta R, Capper D, et al. Spatial transcriptome analysis reveals notch pathway-associated prognostic markers in IDH1 wild-type glioblastoma involving the subventricular zone. BMC Med. 2016;14(1):170.

36. Vassalli G. Aldehyde dehydrogenases: not just markers, but functional regulators of stem cells. Stem Cells Int 2019;2019:3904645.

37. Feng H, Liu Y, Bian X, Zhou F, Liu Y. ALDH1A3 affects colon cancer in vitro proliferation and invasion depending on CXCR4 status. Br J Cancer. 2018; 118(2):224-32

38. Zhang W, Liu Y, Hu H, Huang H, Bao Z, Yang $P$, et al. ALDH1A3: a marker of Mesenchymal phenotype in Gliomas associated with cell invasion. PLoS One. 2015;10(11):e0142856

39. Qiu XX, Wang CH, Lin ZX, You N, Wang XF, Chen YP, et al. Correlation of high delta-like ligand 4 expression with peritumoral brain edema and its prediction of poor prognosis in patients with primary high-grade gliomas. J Neurosurg. 2015;123(6):1578-85.

40. Wu CX, Lin GS, Lin ZX, Zhang JD, Liu SY, Zhou CF. Peritumoral edema shown by MRI predicts poor clinical outcome in glioblastoma. World J Surg Oncol. 2015:13:97.

41. Hegi ME, Diserens AC, Gorlia T, Hamou MF, de Tribolet N, Weller M, et al. MGMT gene silencing and benefit from temozolomide in glioblastoma. $\mathrm{N}$ Engl J Med. 2005;352(10):997-1003.

42. Gilbert MR, Wang M, Aldape KD, Stupp R, Hegi ME, Jaeckle KA, et al. Dosedense temozolomide for newly diagnosed glioblastoma: a randomized phase III clinical trial. J Clin Oncol. 2013:31(32):4085-91.

43. Brell M, Tortosa A, Verger E, Gil JM, Viñolas N, Villá S, et al. Prognostic significance of O6-methylguanine-DNA methyltransferase determined by promoter hypermethylation and immunohistochemical expression in anaplastic gliomas. Clin Cancer Res. 2005;11(14):5167-74.

44. Zawlik I, Vaccarella S, Kita D, Mittelbronn M, Franceschi S, Ohgaki H. Promoter methylation and polymorphisms of the MGMT gene in glioblastomas: a population-based study. Neuroepidemiology. 2009;32(1): 21-9.

45. Ohmura-Kakutani H, Akiyama K, Maishi N, Ohga N, Hida Y, Kawamoto T, et al. Identification of tumor endothelial cells with high aldehyde dehydrogenase activity and a highly angiogenic phenotype. PLoS One. 2014;9(12):e113910.

46. Hida K, Maishi N, Akiyama K, Ohmura-Kakutani H, Torii C, Ohga N, et al. Tumor endothelial cells with high aldehyde dehydrogenase activity show drug resistance. Cancer Sci. 2017;108(11):2195-203.

47. D'Alessio A, Proietti G, Sica G, Scicchitano BM. Pathological and Molecular Features of Glioblastoma and Its Peritumoral Tissue. Cancers (Basel). 2019;11(4).

48. Darmanis S, Sloan SA, Croote D, Mignardi M, Chernikova S, Samghababi $P$, et al. Single-cell RNA-Seq analysis of infiltrating neoplastic cells at the migrating front of human Glioblastoma. Cell Rep. 2017;21(5):1399-410.

49. Charles NA, Holland EC, Gilbertson R, Glass R, Kettenmann H. The brain tumor microenvironment. Glia. 2011;59(8):1169-80.

50. Yao Y, Li N. MIR600HG suppresses metastasis and enhances oxaliplatin chemosensitivity by targeting ALDH1A3 in colorectal cancer. Biosci Rep. 2020;40(4)

51. Pan M, Li M, You C, Zhao F, Guo M, Xu H, et al. Inhibition of breast cancer growth via miR-7 suppressing ALDH1A3 activity concomitant with decreasing breast cancer stem cell subpopulation. J Cell Physiol. 2020; 235(2):1405-16.

52. Cheng P, Wang J, Waghmare I, Sartini S, Coviello V, Zhang Z, et al. FOXD1-ALDH1A3 signaling is a determinant for the self-renewal and Tumorigenicity of Mesenchymal Glioma stem cells. Cancer Res. 2016; 76(24):7219-30.

53. Sneha S, Nagare RP, Sidhanth C, Krishnapriya S, Garg M, Ramachandran B, et al. The hedgehog pathway regulates cancer stem cells in serous adenocarcinoma of the ovary. Cell Oncol (Dordr). 2020. https://doi.org/10.1 007/s13402-020-00504-w.

54. Wong ALA, Hirpara JL, Pervaiz S, Eu JQ, Sethi G, Goh BC. Do STAT3 inhibitors have potential in the future for cancer therapy? Expert Opin Investig Drugs. 2017;26(8):883-7.

55. Caldenhoven E, van Dijk TB, Solari R, Armstrong J, Raaijmakers JA, Lammers JW, et al. STAT3beta, a splice variant of transcription factor STAT3, is a dominant negative regulator of transcription. J Biol Chem. 1996;271(22): $13221-7$.

\section{Publisher's Note}

Springer Nature remains neutral with regard to jurisdictional claims in published maps and institutional affiliations.
Ready to submit your research? Choose BMC and benefit from:

- fast, convenient online submission

- thorough peer review by experienced researchers in your field

- rapid publication on acceptance

- support for research data, including large and complex data types

- gold Open Access which fosters wider collaboration and increased citations

- maximum visibility for your research: over $100 \mathrm{M}$ website views per year

At BMC, research is always in progress.

Learn more biomedcentral.com/submissions 